\title{
Practical Disk Diffusion Test for Detecting Group B Streptococcus with Reduced Penicillin Susceptibility ${ }^{\nabla}$
}

\author{
Kouji Kimura, Jun-ichi Wachino, Hiroshi Kurokawa, Satowa Suzuki, Kunikazu Yamane, \\ Naohiro Shibata, and Yoshichika Arakawa*
}

Department of Bacteriology II, National Institute of Infectious Disease, Tokyo 208-0011, Japan

Received 25 October 2008/Returned for modification 6 December 2008/Accepted 30 September 2009

\begin{abstract}
Although group B streptococcus (GBS) has been considered to be uniformly susceptible to $\beta$-lactams, the presence of GBS with reduced penicillin susceptibility (PRGBS) was recently confirmed genetically. We developed a feasible and reliable method for screening PRGBS in clinical microbiology laboratories using a combination of ceftibuten, oxacillin, and ceftizoxime disks.
\end{abstract}

Streptcoccus agalactiae (group B streptococcus [GBS]) is a leading cause of neonatal sepsis and meningitis and is also an important pathogen for elderly people and those suffering from underlying medical disorders $(1,5,7,11)$. GBS results in the highest mortality and morbidity if it causes invasive infections in neonates, including very-low-birth-weight infants (6, $10,12)$. About $5 \%$ of GBS-infected infants die, and if they survive, they often suffer from severe neurological sequelae, such as mental retardation and vision and/or auditory disabilities (2), but development of GBS vaccines is still under investigation (8). Penicillins are the first-line agents in the treatment of GBS infections because all clinical GBS isolates have been considered to be uniformly susceptible to $\beta$-lactams, including penicillins $(2,3)$. However, we have recently identified and molecularly characterized several clinical GBS isolates demonstrating reduced penicillin susceptibility (PRGBS) through acquisition of multiple mutations in the penicillin-binding protein 2X (pbp2x) gene (9), and similar PRGBS isolates were recently reported in the United States (4). PRGBS isolates were indeed confirmed to be nonsusceptible to penicillin $G$ (PCG) by the agar dilution method, but this PCG nonsusceptibility was not apparent even if the PCG disk diffusion method was performed in accordance with the recommendations of the CLSI (Clinical and Laboratory Standards Institute) (3). Here we developed, therefore, a feasible and practical new disk test method for discriminating PRGBS from the clinically isolated GBS using three disks containing ceftibuten, oxacillin, and ceftizoxime, respectively.

Forty-eight clinical isolates were identified as GBS using a streptococcus grouping kit (Slidex Strepto [bioMerieux, Marcy l'Etoile, France] and Streptex [Mitsubishi Chemical Medience Corporation, Tokyo, Japan]). Streptococcus agalactiae ATCC BAA-611 and ATCC 12403 were used as the reference strains in bacteriological identification. The MICs of PCG for two ATCC standard strains and clinical isolates were determined by the agar dilution method recommended by the CLSI. Strep-

\footnotetext{
* Corresponding author. Mailing address: Department of Bacteriology II, National Institute of Infectious Disease, 4-7-1 Gakuen MusashiMurayama, Tokyo 208-0011, Japan. Phone: 81 (42) 561-0771. Fax: 81 (42) 561-7173. E-mail: yarakawa@nih.go.jp.

${ }^{\nabla}$ Published ahead of print on 7 October 2009.
}

tococcus pneumoniae 49619 was used for validation of the MIC measurements. The disk diffusion method was performed as recommended by the CLSI in evaluation of the applicability of each $\beta$-lactam disk for discrimination of PRGBS strains from penicillin-susceptible strains.

At first, the MICs of PCG for 2 ATCC strains and 48 clinical GBS isolates were measured by the CLSI standard agar dilution methods, and 34 strains, including 2 reference strains, proved susceptible to PCG (MIC, $\leq 0.12 \mu \mathrm{g} / \mathrm{ml}$ ), whereas the remaining 16 clinical isolates were nonsusceptible to PCG (MIC, $>0.12 \mu \mathrm{g} / \mathrm{ml}$ ) (Fig. 1A and Table 1). The highest PCG MIC for such PCG-nonsusceptible GBS strains was $1 \mu \mathrm{g} / \mathrm{ml}$.

In the next step, the nucleotide sequences of the PBP2X genes of all GBS strains used in this investigation were determined, and all 34 penicillin-susceptible GBS (PSGBS) strains were found to harbor neither V405A nor Q557E substitutions in PBP2X, which are conserved among PBP2Xs of almost all PRGBS strains. Among the 16 PRGBS isolates, 15 isolates harbored the PRGBS-specific mutations in PBP2X genes that cause V405A and/or Q557E substitutions in PBP2X, but no such substitution was found in one PRGBS strain, B7, which harbored multiple substitutions in PBP2X other than V405A and Q557E (Table 1), as reported previously (9).

To validate the PCG disk for screening PRGBS, we applied the standard disk diffusion method for GBS with PCG disks in accordance with the CLSI recommendation for streptococci other than pneumococci. For each isolate, the PCG MIC determined by the agar dilution method and the diameter of the growth-inhibitory zone measured by the standard disk diffusion method were plotted on a scatter diagram (Fig. 1A). In all isolates tested, the growth-inhibitory zones around the PCG disk were $>24 \mathrm{~mm}$ (CLSI susceptibility criteria). Thus, it seemed very difficult to exactly discriminate all of the PRGBS from PSGBS by the CLSI standard disk diffusion method using only a PCG disk.

We previously reported that PRGBS showed reduced susceptibility not only to PCG but also to oxacillin and ceftizoxime (9). Thus, we examined the applicability of $44 \beta$-lactam disks commercially available in Japan for detection of PRGBS (Table 2) and found that the ceftibuten disk was also applicable for screening PRGBS.

To evaluate the sensitivity and specificity of the disk diffu- 

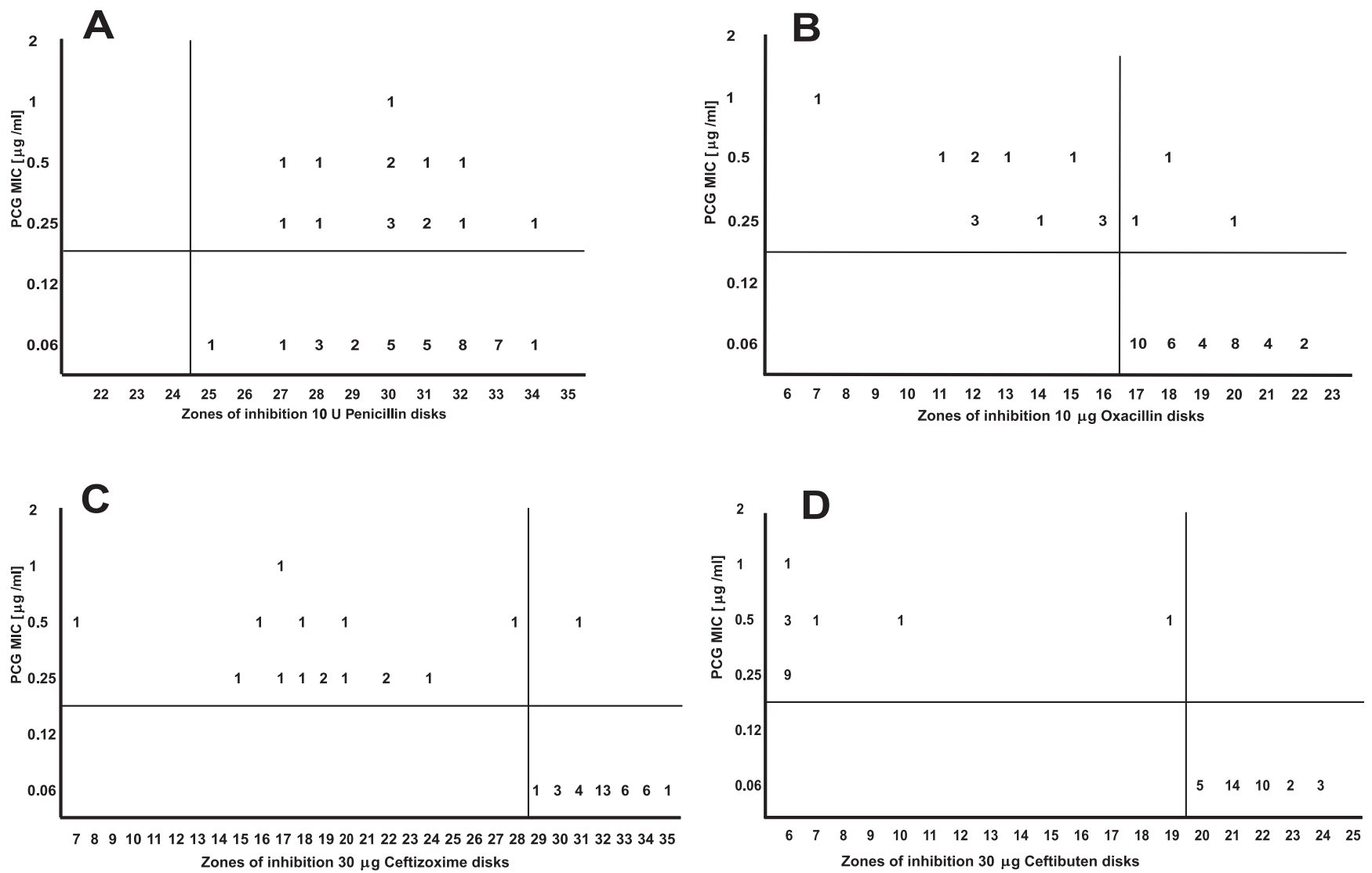

FIG. 1. Scatter diagram of MICs and the sizes of the growth-inhibitory zone. PCG MICs determined by the CLSI agar dilution method and diameters of the growth-inhibitory zone measured by CLSI-recommended standard disk diffusion method are plotted using a PCG disk (A). MICs determined by the agar dilution method and the diameters of growth inhibitory zone measured by the CLSI-recommended standard disk diffusion method are plotted using an oxacillin disk (B), ceftizoxime disk (C), and ceftibuten disk (D). The numbers in the scatter diagram indicate the number of clinical isolates in each intersection.

sion method, we performed the standard disk diffusion method of CLSI using oxacillin, ceftizoxime, and ceftibuten disks (Fig. $1 \mathrm{~B}, \mathrm{C}$, and D). Because the CLSI has not determined the cutoff values for susceptible criteria in these three disks, we set the provisional cutoff values for "reduced susceptible" criteria in the three disks using the smallest-diameter values of susceptible strains: e.g., oxacillin, $<17 \mathrm{~mm}$; ceftizoxime, $<29 \mathrm{~mm}$; and ceftibuten, $<20 \mathrm{~mm}$. Under this condition, the sensitivities of

TABLE 1. MICs of PCG for 16 strains of PRGBS, amino acid substitutions in PBP2X of PRGBS, and the diameters of growth-inhibitory zones around oxacillin, ceftizoxime, and ceftibuten disks

\begin{tabular}{|c|c|c|c|c|c|}
\hline \multirow{2}{*}{ Strain } & \multirow{2}{*}{$\begin{array}{l}\mathrm{MIC}(\mu \mathrm{g} / \mathrm{ml}) \\
\text { of PCG }\end{array}$} & \multirow{2}{*}{ Amino acid substitutions in PBP2X } & \multicolumn{3}{|c|}{ Diam $(\mathrm{mm})$ of growth-inhibitory zone with: } \\
\hline & & & Oxacillin & Ceftizoxime & Ceftibuten \\
\hline B1 & 0.5 & M349I, I377V, F399I, P445S, T555S, Q557E & 13 & 7 & 6 \\
\hline B6 & 0.25 & $\mathrm{E} 411 \mathrm{~K}, \mathrm{~T} 555 \mathrm{~S}, \mathrm{Q} 557 \mathrm{E}$ & 12 & 15 & 6 \\
\hline B7 & 0.25 & I377V, T394A, G398A & 16 & 22 & 6 \\
\hline $\mathrm{B} 8$ & 0.25 & I377V, F395L, P396T, V405A, R433H, H438Y, G648A & 16 & 17 & 6 \\
\hline $\mathrm{B} 10$ & 0.5 & G526R, Q557E, S726L & 15 & 16 & 7 \\
\hline B12 & 0.25 & G526R, Q557E, S726L & 14 & 19 & 6 \\
\hline B40 & 0.5 & P396S, G526R, Q557E, S726L & 11 & 20 & 6 \\
\hline B60 & 0.25 & I377V, T394A, G398A, Q557E & 16 & 22 & 6 \\
\hline B68 & 0.5 & A514V, Q557E & 18 & 28 & 10 \\
\hline B502 & 0.5 & I377V, F395L, V405A, R433H, H438Y, G648A & 12 & 18 & 6 \\
\hline B503 & 0.25 & I377V, F395L, V405A, R433H, H438Y, G648A & 20 & 24 & 6 \\
\hline B513 & 1 & A400V, V405A, Q557E & 7 & 17 & 6 \\
\hline B514 & 0.25 & I377V, F395L, V405A, R433H, H438Y, G648A & 12 & 19 & 6 \\
\hline B516 & 0.25 & I377V, F395L, V405A, R433H, H438Y, G648A & 17 & 18 & 6 \\
\hline M16 & 0.5 & A514V, Q557E & 12 & 31 & 19 \\
\hline M19 & 0.25 & I377V, F395L, V405A, R433H, H438Y, G648A & 12 & 20 & 6 \\
\hline
\end{tabular}


TABLE 2. Antibiotic concentrations of 44 Kirby-Bauer disks used in the applicability check for detecting PRGBS

\begin{tabular}{|c|c|}
\hline Antibiotic & Concn \\
\hline \multicolumn{2}{|l|}{ PCG.. } \\
\hline \multicolumn{2}{|l|}{ Oxacillin...... } \\
\hline \multicolumn{2}{|l|}{ Ampicillin... } \\
\hline \multicolumn{2}{|l|}{ Amoxicillin ... } \\
\hline \multicolumn{2}{|l|}{ Aspoxicillin... } \\
\hline \multicolumn{2}{|c|}{ 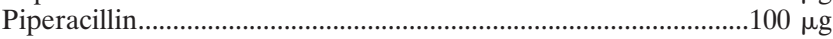 } \\
\hline \multicolumn{2}{|c|}{ 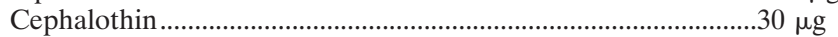 } \\
\hline \multicolumn{2}{|c|}{$\begin{array}{l}\text { Cephalothin } \\
\text { Cefazolin }\end{array}$} \\
\hline \multicolumn{2}{|c|}{ 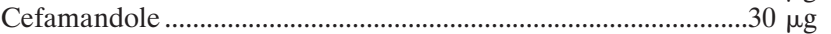 } \\
\hline \multicolumn{2}{|c|}{ 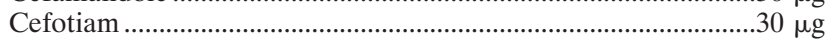 } \\
\hline \multicolumn{2}{|c|}{ 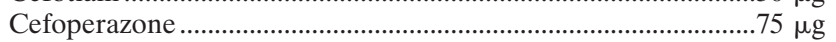 } \\
\hline \multicolumn{2}{|c|}{ Cefuroxime } \\
\hline \multicolumn{2}{|c|}{ Cefotaxime } \\
\hline \multicolumn{2}{|c|}{$\begin{array}{l}\text { Cefotaxime } \\
\text { Ceftizoxime }\end{array}$} \\
\hline \multicolumn{2}{|c|}{ Cefmenoxime } \\
\hline \multicolumn{2}{|c|}{ 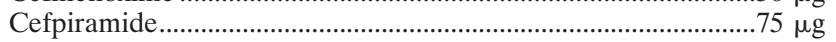 } \\
\hline \multicolumn{2}{|c|}{ 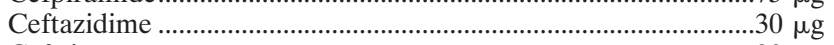 } \\
\hline \multicolumn{2}{|c|}{ 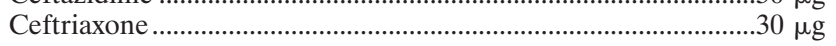 } \\
\hline \\
\hline \multicolumn{2}{|c|}{ 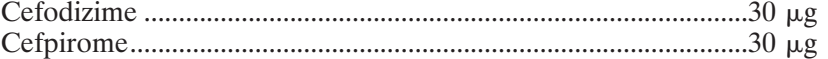 } \\
\hline \multicolumn{2}{|c|}{ Cefepime } \\
\hline \multicolumn{2}{|c|}{ 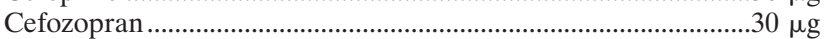 } \\
\hline \multicolumn{2}{|c|}{ Cefsulodin } \\
\hline \multicolumn{2}{|c|}{ 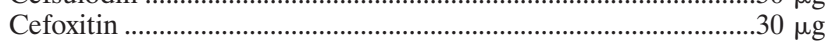 } \\
\hline \multicolumn{2}{|c|}{ 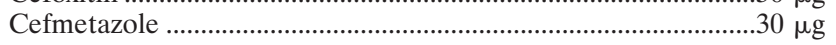 } \\
\hline \multicolumn{2}{|c|}{ Cefotetan } \\
\hline Cefbuperazo & $.75 \mu \mathrm{g}$ \\
\hline Cefminox ..... & $\ldots . .30 \mu \mathrm{g}$ \\
\hline Cephalexin. & $\ldots 30 \mu \mathrm{g}$ \\
\hline Cefaclor .... & $\ldots . .30 \mu \mathrm{g}$ \\
\hline Cefixime........ & $\ldots 5 \mu \mathrm{g}$ \\
\hline Ceftibuten.... &. $.30 \mu \mathrm{g}$ \\
\hline Cefdınır..... & $.5 \mu \mathrm{g}$ \\
\hline Cefpodoxime & $\ldots 10 \mu \mathrm{g}$ \\
\hline Cefteram...... & $\ldots 10 \mu \mathrm{g}$ \\
\hline Cefcapene... & $\ldots . .5 \mu \mathrm{g}$ \\
\hline Cefditoren ... &. $.5 \mu \mathrm{g}$ \\
\hline Moxalactam. & $\ldots . . .30 \mu \mathrm{g}$ \\
\hline Flomoxef....... & $\ldots 30 \mu \mathrm{g}$ \\
\hline Imipenem..... & $\ldots 10 \mu \mathrm{g}$ \\
\hline Panipenem... & $\ldots 10 \mu \mathrm{g}$ \\
\hline Meropenem. & \\
\hline Aztreonam... & $\ldots . .30 \mu \mathrm{g}$ \\
\hline Carumonam. & $\ldots . .30 \mu \mathrm{g}$ \\
\hline
\end{tabular}

these disks were 13/16 (81\%), 15/16 (94\%), and 16/16 (100\%), respectively, and the respective specificities were 34/37 (92\%), $34 / 35(97 \%)$, and $34 / 34(100 \%)$. Thus, the sensitivity and specificity of the CLSI standard disk method using oxacillin, ceftizoxime, and ceftibuten disks were confirmed to be fully applicable for discrimination of PRGBS from the clinical GBS isolates. Moreover, all of the clinical PRGBS isolates were detected by at least one of the three disks. Therefore, the combination of the three disks could be expected to successfully distinguish all of the PRGBS from PSGBS (Table 3 ) in the routine work of clinical microbiology laboratories.

Indeed, one PRGBS isolate showed a 19-mm growth-inhibitory zone around the ceftibuten disk (Fig. 1D and Table 1), and this strain might well be misclassified as PSGBS. However, the results from the other disk containing oxacillin would complement exact detection of PRGBS. The PRGBS detection method developed in this investigation using ceftibuten, as well
TABLE 3. Specificity and sensitivity of each $\beta$-lactam disk used in the disk diffusion methods

\begin{tabular}{llc}
\hline \multirow{2}{*}{ Disk } & \multicolumn{2}{c}{ No. of isolates/total $(\%)^{a}$} \\
\cline { 2 - 3 } & \multicolumn{1}{c}{ Specificity } & Sensitivity \\
\hline Penicillin & $34 / 50(68)$ & $0 / 16(0)$ \\
Oxacillin & $34 / 37(92)$ & $13 / 16(81)$ \\
Ceftizoxime & $34 / 35(97)$ & $15 / 16(94)$ \\
Ceftibuten & $34 / 34(100)$ & $16 / 16(100)$ \\
Combination & $34 / 34(100)$ & $16 / 16(100)$ \\
\hline
\end{tabular}

${ }^{a}$ Denominators of specificity values are the numbers of isolates with a growthinhibitory zone diameter around each disk above the tentative cutoff value. Denominators of sensitivity values are the numbers of PRGBS isolates determined by PCG MICs.

as oxacillin and ceftizoxime disks, would promise high specificity and sensitivity without necessitating any expensive or special equipment. This method, therefore, could come into wide use for detection of PRGBS in the daily antimicrobial susceptibility testing of GBS after its validation by multiple reference laboratories. Due to the lack of exact detection test methods for PRGBS, no confirmed case of penicillin treatment failure has been reported in GBS infections to date, and no clinical significance of PRGBS has so far been evaluated. Indeed, the PRGBS isolates were identified by measurement of MICs using the agar dilution method without regard for penicillin treatment failure. Moreover, it seems very difficult without case-controlled analyses to conclude whether the antibiotic treatment failure in the GBS infection cases is mainly due to bacterial penicillin nonsusceptibility or depends on a deteriorated ability to defend against microbial infections of the host. The new test method reported here offers a promising, easy, and reliable way to detect PRGBS and thus promote case analyses of infections with PRGBS in the future.

This study was supported by H18-Shinkou-011, from the Ministry of Health, Labor and Welfare, Japan. K. Kimura was a fellow of the Japan Health Sciences Foundation.

We thank Kumiko Kai and Yoshie Taki for technical assistance and Miroku Medical Laboratory, Co., Ltd., for donating clinical isolates of GBS.

\section{REFERENCES}

1. Baker, C. J. 2000. Group B streptococcal infections. p. 222-237. In D. L. Stevens and E. L. Kaplan (ed.), Streptococcal infections. Clinical aspects, microbiology, and molecular pathogenesis. Oxford University Press, Oxford, England.

2. Centers for Disease Control and Prevention. 2002. Prevention of perinatal group B streptococcal disease. MMWR Morb. Mortal. Wkly. Rep. 51:1-22.

3. Clinical and Laboratory Standards Institute. 2009. Performance standards for antimicrobial susceptibility testing. Nineteenth informational supplement M100-S19. Clinical and Laboratory Standards Institute, Wayne, PA.

4. Dahesh, S., M. E. Hensler, N. M. Van Sorge, R. E. Gertz, Jr., S. Schrag, V. Nizet, and B. W. Beall. 2008. Point mutation in the group B streptococcal pbp $2 x$ gene conferring decreased susceptibility to $\beta$-lactam antibiotics. Antimicrob. Agents Chemother. 52:2915-2918.

5. Farley, M. M., R. C. Harvey, T. Stull, J. D. Smith, A. Schuchat, J. D. Wenger, and D. S. Stephens. 1993. A population-based assessment of invasive disease due to group B streptococcus in nonpregnant adults. N. Engl. J. Med. 328: $1807-1811$.

6. Heath, P. T., G. Balfour, A. M. Weisner, A. Efstratiou, T. L. Lamagni, H. Tighe, L. A. O'Connell, M. Cafferkey, N. Q. Verlander, A. Nicoll, A. C. McCartney, and the PHLS Group B Streptococcus Working Group. 2004. Group B streptococcal disease in UK and Irish infants younger than 90 days. Lancet 363:292-294.

7. Jackson, L. A., R. Hilsdon, M. M. Farley, L. H. Harrison, A. L. Reingold, 
B. D. Plikaytis, J. D. Wenger, and A. Schuchat. 1995. Risk factors for group B streptococcal disease in adults. Ann. Intern. Med. 123:415-420.

8. Johri, A. K., L. C. Paoletti, P. Glaser, M. Dua, P. K. Sharma, G. Grandi, and R. Rappuoli. 2006. Group B streptococcus: global incidence and vaccine development. Nat. Rev. Microbiol. 4:932-942.

9. Kimura, K., S. Suzuki, J. Wachino, H. Kurokawa, K. Yamane, N. Shibata, N. Nagano, H. Kato, K. Shibayama, and Y. Arakawa. 2008. First molecular characterization of group B streptococci with reduced penicillin susceptibility. Antimicrob. Agents Chemother. 52:2890-2897.
10. Schuchat, A. 1998. Epidemiology of group B streptococcal disease in the United States: shifting paradigms. Clin. Microbiol. Rev. 11:497-513.

11. Schuchat, A. 1999. Group B streptococcus. Lancet 353:51-56.

12. Stoll, B. J., N. Hansen, A. A. Fanaroff, L. L. Wright, W. A. Carlo, R. A. Ehrenkranz, J. A. Lemons, E. F. Donovan, A. R. Stark, J. E. Tyson, W. Oh, C. R. Bauer, S. B. Korones, S. Shankaran, A. R. Laptook, D. K Stevenson, L. A. Papile, and W. K. Poole. 2002. Changes in pathogens causing early-onset sepsis in very-low-birth-weight infants. N. Engl. J. Med. 347:240-247. 